\title{
Symmetry and chirality in crystals
}

\author{
Massimo Nespolo and Amani Hind Benahsene
}

J. Appl. Cryst. (2021). 54, 1594-1599

\section{IU IUCr Journals CRYSTALLOGRAPHY JOURNALS ONLINE}

Author(s) of this article may load this reprint on their own web site or institutional repository provided that this cover page is retained. Republication of this article or its storage in electronic databases other than as specified above is not permitted without prior permission in writing from the IUCr.

For further information see https://journals.iucr.org/services/authorrights.html 


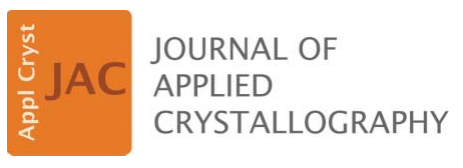

ISSN 1600-5767

Received 23 July 2021

Accepted 3 September 2021

Edited by S. Moggach, The University of Western Australia, Australia

Keywords: chirality; handedness; kryptoracemate; racemic; scalemic.

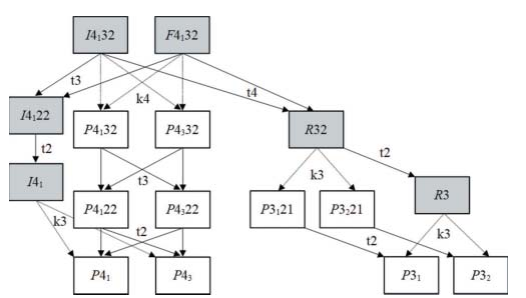
of chiral molecules in which the two enantiomers occur in different amounts.

Sohncke type of space group: a space group containing only operations of the first kind.

$Z$ : number of unit formulae (number of molecules for molecular crystals) in the unit cell.

$Z^{\prime}$ : number of unit formulae (number of molecules for molecular crystals) in the asymmetric unit.

\section{Glossary of terms used in this article}

Achiral: the opposite of chiral.

Chiral: describing an object which presents chirality.

Chirality: the geometric property of a rigid object of being non-superposable on its mirror image; such an object has no symmetry operations of the second kind.

Chiral type of space group: a space group containing screw axes of type $n_{p}$ or $n_{n-p}$ with $p \neq n / 2$ but not both of them.

Conglomerate: a crystal in which the two enantiomers occur in physically separated domains; depending on whether the volume spanned by the two types of domains is equal or not, the conglomerate is racemic or scalemic.

Enantiomers: same as enantiomorphs but related to molecules. Enantiomorphs: two chiral objects which are the mirror images of one another.

Handedness: the property differentiating two enantiomorphs or enantiomers (being 'left' and 'right'). Also known as chirality sense.

Isometry: a distance-preserving transformation.

Kryptoracemate: a racemic structure crystallizing in a Sohncke type of space group.

Operation of the first kind: a handedness-preserving isometry. Operation of the second kind: a handedness-reversing isometry.

Racemic: describing a mixture, solution or crystal of chiral molecules in which both enantiomers form in equal amounts. Scalemic: describing a non-racemic mixture, solution or crystal 


\section{Introduction}

The International Union of Pure and Applied Chemistry defines chirality as 'the geometric property of a rigid object (or spatial arrangement of points or atoms) of being non-superposable on its mirror image; such an object has no symmetry elements of the second kind ${ }^{1}$, (IUPAC, 1997). The property of being 'non-superimposable' means that the object and its mirror image are not directly congruent, i.e. they cannot be mapped onto one another by an isometry of the first kind. An object that presents this property is chiral; its opposite is achiral. A chiral object and its mirror image are called enantiomorphs or, when referring to molecules, enantiomers. Two enantiomorphs are said to have opposite handedness ('left' and 'right'), or chirality sense. Because the term 'chirality sense' is often incorrectly shortened to 'chirality', in the following we only use the term 'handedness' to indicate the property that distinguishes enantiomorphs (enantiomers). A sample in which all molecules have (within limits of detection) the same handedness is called enantiomerically pure, or enantiopure for short. Following Heathcock (1991) and Flack (2003), we reject the term 'homochiral', adopted by some authors.

Two enantiomers have similar physical and chemical properties. If the synthesis is from an isotropic phase and in the absence of any chiral object, which could favour one enantiomer over the other, the result should be a racemic mixture, in which both enantiomers form in equal amounts. Montoya et al. (2018) have shown that this is not always the case.

We are interested in the symmetry conditions under which a crystal structure can or cannot be chiral. The problem has been analysed in detail by Flack (2003). However, the case of kryptoracemic compounds, formerly known as "false conglomerates' (Bishop \& Scudder, 2009), i.e. racemic structures crystallizing in a Sohncke type of space group, was not addressed. Although the term kryptoracemate was first used in 1995 (Bernal, 1995) and a report of a kryptoracemic compound was published the following year (Morales \& Fronczek, 1996), technical difficulties related to the lack of software capable of identifying the absolute configuration of asymmetric atoms present in molecular models deposited in databases have delayed a consistent and systematic analysis. Moreover, the case of enantioenriched scalemic compounds was not explicitly addressed. In this article we aim to propose a general classification scheme relating the chirality of molecules and the chirality of the crystal structure they may form.

\section{Types of space groups in which chiral compounds can or cannot occur}

We briefly recall the classification presented by Nespolo et al. (2018). Chiral structures can crystallize in space groups that contain only operations of the first kind, i.e. handednesspreserving operations. These space groups belong to 65 types

\footnotetext{
1 The statement should be about operations, not elements, of the second kind. First (handedness-preserving) versus second (handedness-reversing) kind applies to operations, not to the elements.
}

called Sohncke space-group types. We emphasize that the condition is necessary but not sufficient: a chiral crystal structure necessarily occurs in a Sohncke type of space group, but not all crystal structures occurring in a Sohncke type of space group are chiral.

Sohncke groups are often incorrectly called 'chiral groups'. Sohncke groups are groups in which a chiral structure can occur, but the groups themselves are not necessarily chiral. Chiral groups are a subset of Sohncke groups and belong to 22 types, occurring in 11 enantiomorphic pairs. These types of space groups contain screw axes $n_{p}$ or $n_{n-p}$ with $p \neq n / 2$ but not both of them. For example, the Sohncke type $P 4_{2}$ (No. 77), for which $p=n / 2$, is not chiral: it does not have an enantiomorphic counterpart, as easily shown by the fact that $P 4_{4-2}=$ $P 4_{2}$. However, two space groups of type $P 4_{1}$ (No. 76) and $P 4_{3}$ (No. 78) are both chiral and constitute a pair of enantiomorphic space groups. The former contains $4_{1}$ screw axes but not $4_{3}$, the latter the opposite. Adding an $I$-centring vector makes the two types of screw axes, $4_{1}$ and $4_{3}$, coexist. The augmented-matrix representation of the $4_{1}$ screw rotation in the conventional coordinate reference of $I 4_{1}$ is

$$
\left(\begin{array}{lll|l}
0 & \overline{1} & 0 & 0 \\
1 & 0 & 0 & \frac{1}{2} \\
0 & 0 & 1 & \frac{1}{4}
\end{array}\right),
$$

which corresponds to a $4_{1}$ screw rotation about an axis located at $\frac{1}{4}, \frac{1}{4}, z$. By adding the $I$ centring, it becomes

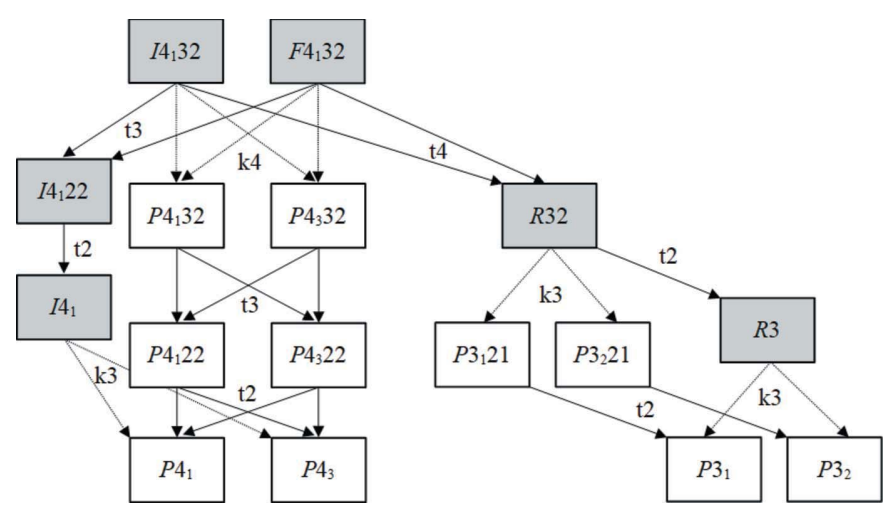

Figure 1

Tree of maximal group-subgroup relations for Sohncke-type space groups containing screw axes $n_{p}$ with $p \neq n / 2$. Grey background: achiral types containing screw axes of opposite handedness whose coexistence is due to the presence of centring vectors $(I, F$ or $R)$. White background: chiral types obtained as translationengleiche ( $\mathrm{t}$ ) subgroups (same translation lattice, but a reduction in point symmetry) or klassengleiche (k) subgroups (same point group but a reduction in the translational symmetry). $\mathrm{t} j$ and $\mathrm{k} j$ indicate, respectively, $\mathrm{t}$ and $\mathrm{k}$ subgroups of index $[j]$. For k subgroups, the coordinate systems of the group and of the subgroup are kept parallel and only loss of centring translations is considered. Ten out of 22 chiral types of groups (five enantiomorphic pairs out of 11) can be obtained in this way. Four other types (two enantiomorphic pairs: $P 3_{1} 12-P 3_{2} 12 ; P 4_{1} 2_{1} 2-P 4_{3} 2_{1} 2$ ) can be obtained through cell enlargement. The remaining eight types (four enantiomorphic pairs: $P 6_{1} 22-P 6_{5} 22$; $\left.P 6_{2} 22-P 6_{4} 22 ; P 6_{1}-P 6_{5} ; P 6_{2}-P 6_{4}\right)$ are hexagonal and cannot be obtained as subgroups of the cubic Sohncke-type space group at the top of the tree. 


$$
\left(\begin{array}{ccc|c}
0 & \overline{1} & 0 & \frac{1}{2} \\
1 & 0 & 0 & 1 \\
0 & 0 & 1 & \frac{3}{4}
\end{array}\right),
$$

which corresponds to a $4_{3}$ screw rotation about an axis located at $\frac{1}{4}, \frac{3}{4}, z$. A space group of type $I 4_{1}($ No. 80$)$ contains both $4_{1}$ and $4_{3}$ screw axes and is therefore not chiral. The same situation is found in six Sohncke types of space group: $I 4_{1} 32$, $F 4_{1} 32$, and their subgroups $I 4_{1} 22, I 4_{1}, R 32$ and $R 3$. In the two cubic and the two tetragonal types of space group, screw axes of type $4_{1}$ and $4_{3}$ coexist owing to the $I$ or $F$ centring; in the two rhombohedral types of space group, screw axes of type $3_{1}$ and $3_{2}$ coexist owing to the $R$ centring. Fig. 1 shows a tree of maximal group-subgroup relations for Sohncke-type space groups containing screw axes $n_{p}$ with $p \neq n / 2$. The grey background corresponds to achiral types containing screw axes of opposite handedness whose coexistence is due to the presence of centring vectors $(I, F$ or $R$ ). The white background indicates chiral types obtained as translationengleiche subgroups or klassengleiche subgroups. For klassengleiche subgroups, the coordinate systems of the group and of the subgroup are kept parallel and only loss of centring translations is considered.

\section{Molecular versus structural chirality}

The relation between the chirality of the molecules and the chirality of the crystal structure they form can be established for ordered crystal structures.

The crystallization of a solution of achiral molecules can result in both a chiral and an achiral crystal structure. Rekis (2020) classified chiral and achiral crystal structures from achiral molecules simply on the basis of whether the space group is or is not of Sohncke type. However, for a crystal structure to be chiral, one needs either the molecules or the molecular packing to be chiral (possibly both). For the molecular packing to be chiral, the absence of isometries of the second kind is not sufficient: the presence of screw axes $n_{p}$ with $p \neq n / 2$ is necessary, which is realized in 28 types of space group: the 22 chiral types of space group and the six types containing screw axes of opposite handedness, shown in grey in Fig. 1(a). As a consequence, an achiral crystal structure from achiral molecules occurs in the remaining $230-28=202$ types of space group.

(1) A racemic solution contains both enantiomers, L (left) and $\mathrm{R}$ (right), in equal amounts. The crystallization from a racemic solution can produce two types of crystal structure (Flack, 2003):

(1.1) a racemic conglomerate, in which the two enantiomers occur in physically separated domains (the volume spanned by the two types of domains is equal);

(1.2) a racemic crystal, in which the two enantiomers are present in equal amounts in the unit cell.

(2) A non-racemic solution of chiral molecules contains both enantiomers, $\mathrm{L}$ and $\mathrm{R}$, in different amounts. Brewster (1992) proposed the term scalemic for this type of solution. The term is not present in the 'Gold Book' (IUPAC, 1997), but
Eliel (1997) adopted it and also divided scalemic solutions into enantiopure (enantiomerically pure) and enantioenriched (having an excess of one enantiomer but not to the exclusion of the other). The crystallization of an enantiopure solution will produce a chiral crystal structure. Its space group will belong to one of the 65 Sohncke types. The crystallization of an enantioenriched solution will follow the same scheme as a racemic solution but with unequal amounts of both enantiomers, leading to

(2.1) a scalemic conglomerate, in which the two enantiomers occur in physically separated domains, spanning a different volume;

(2.2) a scalemic crystal, in which the two enantiomers are present in different amounts in the unit cell.

The terms racemic conglomerate and racemic crystal are well established in the literature, but the corresponding terms for scalemic are much rarer, although not unknown. The first occurrence we could locate of scalemic conglomerate is in an article by Wei et al. (2009), whereas scalemic crystal seems to have been used only recently (Neugebauer et al., 2021).

\subsection{Scalemic and racemic conglomerates}

In a scalemic or racemic conglomerate, the space group of each domain, $\mathcal{G}(\mathrm{L})$ and $\mathcal{G}(\mathrm{R})$ in the following, is of Sohncke type and contains only isometries of the first kind, $\mathrm{f}_{\mathrm{I}}$ in the following. The operation mapping the two types of domain is an isometry of the second kind, $\mathrm{f}_{\mathrm{II}}$ in the following. $\mathcal{G}(\mathrm{L})$ and $\mathcal{G}(\mathrm{R})$ are conjugate under $\mathrm{f}_{\mathrm{II}}$ :

$$
\mathrm{R}=\mathrm{f}_{\mathrm{II}} \mathrm{L} ; \quad \mathcal{G}(\mathrm{R})=\mathrm{f}_{\mathrm{II}} \mathcal{G}(\mathrm{L}) \mathrm{f}_{\mathrm{II}}^{-1} .
$$

Any isometry of the second kind can be expressed as the product of an inversion and an isometry of the first kind:

$$
\begin{gathered}
\mathrm{f}_{\mathrm{II}}=\mathrm{f}_{\mathrm{I}} \overline{1} ; \quad \mathrm{R}=\mathrm{f}_{\mathrm{II}} \mathrm{L}=\mathrm{f}_{\mathrm{I}} \overline{\mathrm{L}} \mathrm{L}=\mathrm{f}_{\mathrm{I}} Я, \\
Я=\overline{1} \mathrm{~L}=\mathrm{f}_{\mathrm{I}}^{-1} \mathrm{R},
\end{gathered}
$$

where $Я$ has the same handedness as $\mathrm{R}$. The proportion of the two enantiomorphs $\mathrm{L}$ and $\boldsymbol{Я}$ present in the conglomerate is measured by the Flack parameter $x$ (Flack, 1983). If $x=0.5$, the two types of domain span the same volume and the conglomerate is racemic; if $x$ deviates from 0.5 in a statistically significant way (i.e. outside three standard uncertainties on $x$ ), the two types of domain span different volumes and the conglomerate is scalemic.

The conjugation relation (1) can be rewritten as follows:

$$
\mathcal{G}(\mathrm{R})=\left(\mathrm{f}_{\mathrm{I}} \overline{1}\right) \mathcal{G}(\mathrm{L})\left(\mathrm{f}_{\mathrm{I}} \overline{1}\right)^{-1}=\mathrm{f}_{\mathrm{I}}[\overline{1} \mathcal{G}(\mathrm{L}) \overline{1}] \mathrm{f}_{\mathrm{I}}^{-1} .
$$

While $\mathrm{f}_{\mathrm{I}}$ does not influence the handedness of a screw axis, the inversion does. If $g$ is an operation of $\mathcal{G}$, the conjugation $\overline{1} g \overline{1}$ has the same linear (matrix) part as $g$ while the translation (vector) part is inverted. The result is that the screw axes in $\mathcal{G}(\mathrm{R})$ have opposite handedness with respect to those in $\mathcal{G}(\mathrm{L})$. It follows that

(1) if $\mathcal{G}(\mathrm{L})$ is not chiral, then $\mathcal{G}(\mathrm{R})$ is of the same type as $\mathcal{G}(\mathrm{L})$, i.e. both domains have the same type of space group;

(2) if $\mathcal{G}(\mathrm{L})$ is chiral, then $\mathcal{G}(\mathrm{R})$ is its enantiomorphic counterpart. 
The result above is easily understood considering that a Sohncke achiral group can be of three types:

(1) a group that does not contain screw axes (e.g. P2);

(2) a group whose screw axes do not possess handedness, i.e. $n_{p}$ with $p=n / 2$ (e.g. $\left.P 2_{1}, P 4_{2}, P 6_{3}\right)$;

(3) a group that contains screw axes of opposite handedness because of a centring translation (grey background in Fig. 1).

\subsection{Scalemic and racemic crystals}

A racemic crystal (sensu stricto, i.e. exclusive of kryptoracemates) contains both enantiomers in the asymmetric unit, and these are related by an isometry of the second kind which is a operation of the space group. Therefore, racemic crystals occur only in non-Sohncke types of space groups.

An enantiopure scalemic crystal contains only one type of enantiomer, which excludes the presence of isometries of the second kind. An enantioenriched scalemic crystal contains both types of enantiomers but in different amounts. If its space group contained isometries of the second kind, then the ratio of the two enantiomers would be 50:50. Therefore, the space group of a scalemic crystal, whether enantiopure or enatioenriched, is necessarily of Sohncke type.

In his survey of the Cambridge Structural Database (CSD; http://www.ccdc.cam.ac.uk), Rekis (2020) found that $50.5 \%$ of chiral compounds occurred as racemic crystals, about $44.1 \%$ as enantiopure scalemic crystals and about $5.4 \%$ as racemic conglomerates. Kryptoracemates were rare (313 occurrences) and scalemic compounds even rarer (13 occurrences). Rekis suggested, however, that many more scalemic compounds probably exist, but they are difficult to discover on a simple database exploration. Identifying them requires the analysis of crystallization studies.

Clevers \& Coquerel (2020) reported that crystallization from racemic solutions results in racemic crystals in $90-95 \%$ of cases, racemic conglomerates in $5-10 \%$ of cases, solid solutions in $1-2 \%$ of cases and scalemic enantioenriched crystals in less than $1 \%$ of cases.

\subsection{Hunting for kryptoracemates}

It has long been considered that the space group of a racemic crystal necessarily contains operations of the second kind, relating the two enantiomers, and is therefore not of Sohncke type. This restriction was lifted with the discovery of kryptoracemates, i.e. racemic structures crystallizing in a Sohncke-type space group (Bernal, 1995). To explain their existence, the condition $Z^{\prime}>1$ was introduced when surveys of the CSD were run to identify the occurrence of kryptoracemates (Fábián \& Brock, 2010; Bernal \& Watkins, 2015; Clevers \& Coquerel, 2020). It was assumed that the two enantiomers must occur in the asymmetric unit and be approximately related by an isometry of the second kind, often an inversion. This mapping being only approximate, the corresponding operation is only a pseudo-symmetry operation, sometimes incorrectly called 'non-crystallographic' (Clevers \& Coquerel, 2020) instead of partial or pseudo or pseudo-partial (Nespolo et al., 2008). This operation does not
Table 1

Atomic displacements in $\AA$ necessary to promote the space-group symmetry of $\left[\mathrm{Cr}(\mathrm{bpy})_{3}\right]\left(\mathrm{PF}_{6}\right)_{3}$ and $\left[\mathrm{Rh}(\mathrm{bpy})_{3}\right]\left(\mathrm{PF}_{6}\right)_{3}$ from $R 32$ to $R \overline{3} c$.

Only the heavy-metal atoms are compatible with the supergroup. The displacements of carbon and especially fluorine atoms are too large and incompatible with $R \overline{3} c$ symmetry, contrary to the hypothesis of Bernal \& Watkins (2015). These two structures are therefore kryptoracemates with $Z^{\prime}=$ 1 , showing that the restriction $Z^{\prime}>1$ previously assumed for the occurrence of kryptoracemates is unnecessary.

\begin{tabular}{lll}
\hline Atom & {$\left[\mathrm{Cr}(\mathrm{bpy})_{3}\right]\left(\mathrm{PF}_{6}\right)_{3}$} & {$\left[\mathrm{Rh}(\mathrm{bpy})_{3}\right]\left(\mathrm{PF}_{6}\right)_{3}$} \\
\hline $\mathrm{C}$ & 0.1441 & 0.0828 \\
$\mathrm{~F}$ & 0.2904 & 0.1933 \\
$\mathrm{Cr} / \mathrm{Rh}$ & 0.0000 & 0.0000 \\
$\mathrm{P}$ & 0.0225 & 0.0154 \\
$\mathrm{~N}$ & 0.0910 & 0.0317 \\
\hline
\end{tabular}

appear in the space group, allowing for a racemic compound in a Sohncke-type space group. Well before those surveys, Zorkii (1978) had recognized the existence of molecular crystals characterized by $Z^{\prime}>1$, which he called supersymmetric structures; he, however, focused on those structures in which the independent molecules in the asymmetric unit can be brought to more or less exact superposition by a screw rotation about a direction relating the molecules.

Both Fábián \& Brock (2010) and Bernal \& Watkins (2015) recognized that the restriction to $Z^{\prime}>1$ could exclude kryptoracemic molecules on special positions, e.g. on rotation axes. Fábián \& Brock (2010) found 181 kryptoracemates, of which three were in chiral groups $\left(P 4_{1}, P 4_{1} 2_{1} 2, P 4_{3} 2_{1} 2\right)$. Two occurred in a non-chiral group of type $I 4_{1}$. However, the value of $Z^{\prime}$ reported was 1.5 , indicating that these are not racemic but scalemic enantioenriched crystals; the two enantiomers (LRR ou LLR) were all found on twofold axes.

Bernal \& Watkins (2015) extracted 1412 chiral organometallic crystal structures, among which they identified 26 credible kryptoracemates, in addition to those previously found by Fábián \& Brock (2010). Of these, one occurs in a chiral type of space group (namely, of type $P 4_{1}$ ) and none in space groups containing screw axes of opposite handedness.

Clevers \& Coquerel (2020) processed 393012 organic entries and estimated that racemic crystals constitute 23.8 and $22.2 \%$ of centrosymmetric and achiral non-centrosymmetric organic structures, respectively. Scalemic enantioenriched crystals were very rare (37 out of 393 012). The kryptoracemates represented $0.2 \%$ of the whole database and $0.8 \%$ of the Sohncke-type space groups (incorrectly called 'chiral space groups').

Two isostructural crystals $\left[\mathrm{Cr}(\mathrm{bpy})_{3}\right]\left(\mathrm{PF}_{6}\right)_{3}$ and $\left[\mathrm{Rh}(\mathrm{bpy})_{3}\right]-$ $\left(\mathrm{PF}_{6}\right)_{3}(\mathrm{CSD}$ codes FERYEG and FERYIK, respectively; bpy is $2,2^{\prime}$-bipyridine) were reported by Hauser et al. (1987) in $R 32$ with $Z=6$, i.e. $Z^{\prime}=1$, contrary to the assumption $Z^{\prime}>1$. The two enantiomers are centred on cations located along the threefold axis, i.e. precisely the cases missed by the search criterion $Z^{\prime}>1$. Bernal \& Watkins (2015) included these two structures among the 16 structures showing 'indications of improper space-group selection' and suggested that the correct space group would actually be $R \overline{3} c$, therefore transforming these two kryptoracemates into normal racemates. 
Table 2

Relation between the chirality of molecules and the possible chirality of crystal structures they may form.

\begin{tabular}{|c|c|c|}
\hline & Achiral crystal structure & Chiral crystal structure \\
\hline Achiral molecules & 202 types of space group & $\begin{array}{l}28 \text { types of space group with screw axis } n_{p} \text { where } p \neq \\
n / 2\end{array}$ \\
\hline Chiral molecules, enantiopure scalemic solution & Impossible & 65 Sohncke types of space group \\
\hline Chiral molecules, enantioenriched scalemic solution & $\begin{array}{l}230 \text { types of space group: type I (disordered) solid } \\
\text { solutions }\end{array}$ & $\begin{array}{l}65 \text { Sohncke types of space group: scalemic crystals or } \\
\text { scalemic conglomerates }\end{array}$ \\
\hline Chiral molecules, racemate & $\begin{array}{l}165 \text { non-Sohncke types of space group: racemic } \\
\text { crystals } \\
65 \text { Sohncke types of space group: kryptoracemates }\end{array}$ & $\begin{array}{l}65 \text { Sohncke types of space group: racemic conglom- } \\
\text { erates }\end{array}$ \\
\hline
\end{tabular}

We have run a check for pseudosymmetry using the PSEUDO routine (Capillas et al., 2011) at the Bilbao Crystallographic Server (Aroyo et al., 2006). The atomic displacements from the positions in $R 32$ which would make the structures compatible with $R \overline{3} c$ are shown in Table 1 (the hydrogen atoms were not refined, and we therefore ignore their contribution to pseudosymmetry). The displacements are as large as $0.29 \AA$ for

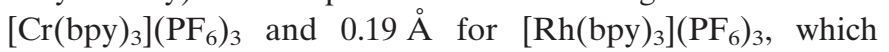
correspond to 23 s.u. (standard uncertainity) and 15 s.u., respectively, in terms of fractional atomic coordinates. Considering the quality of the structure refinement $\left(R_{\text {all }}=\right.$ 0.048 and 0.036 , respectively), we can reasonably exclude a wrong assignment of the space group. Note that in both compounds the metal atoms (either $\mathrm{Cr}$ or $\mathrm{Rh}$ ) are located at Wyckoff positions $3 a$ and $3 b$, which belong to the same Wyckoff set, and correspond to the invariant lattice complex $R$ and $00 \frac{1}{2} R$, respectively, formed by all rhombohedral point lattices (Fischer et al., 1973). The two lattice complexes coalesce in a single lattice complex, symbolized by $00 \frac{1}{4} R c$, in the $6 a$ position of $R \overline{3} c$ : this is why the atomic displacements for the metal atoms in Table 1 are zero. Because the contribution to the diffraction intensities from the heavy-metal atoms is highly significant, the doubt cast between $R 32$ and $R \overline{3} c$ may have arisen because the metal atoms contribute to the diffraction with higher symmetry; the lighter atoms are, however, clearly too far from the positions required by $R \overline{3} c$. These two compounds are therefore examples of kryptoracemates crystallizing in Sohncke groups with $Z^{\prime}=1$, showing that the restriction $Z^{\prime}>1$ on kryptoracemates is unnecessary.

\section{Solid solutions and disordered structures}

From a structural viewpoint, two types of solid solutions have been introduced (Rekis \& Bērziņš, 2018).

(a) In type I solid solutions both enantiomers are disordered in the unit cell, independently of whether the composition is racemic or scalemic.

(b) In type II solid solutions the enantiomers are completely ordered at racemic composition; they correspond to racemic or scalemic crystals as discussed above.

The disordered arrangement of enantiomers in a type I solid solution prevents any restriction on the type of space group (Clevers \& Coquerel, 2020). However, because of the statistical distribution of enantiomers the crystal structure cannot be chiral.

\section{Chirality in non-molecular compounds}

Chirality is by no means an exclusive feature of molecular compounds. Purely inorganic compounds do also occur in opposite enantiomorphs. The most well known example is that of quartz, which occurs in two enantiomorphs, both at low temperature $(\alpha$-quartz) and at high temperature ( $\beta$-quartz) (Glazer, 2018). The handedness of the crystal structure is reflected in the morphology, the two enantiomorphs being distinguishable by the orientation of the $\{11 \overline{2} 1\}$ and $\{51 \overline{6} 1\}$ forms, when present.

The fundamental building unit of a molecular compound is unambiguously identified as the molecule itself. For a purely inorganic compound, a chiral crystal structure can sometimes be obtained from a solution containing achiral reactants. A simple example is the case of $\mathrm{CsCuCl}_{3}$, prepared from an aqueous solution containing $\mathrm{CsCl}$ and $\mathrm{CuCl}_{2}$ by the slow evaporation of a slightly acidified solvent. Right-handed (space-group type $P 6_{1} 22$ ) and left-handed (space-group type $P 6_{5} 22$ ) crystal structures were obtained in this way (Kousaka et al., 2009).

\section{Discussion}

Most but not all chiral crystal structures are formed by molecular compounds. The chirality or achirality of the molecules is reflected in some restrictions on the chirality or achirality of the crystal structures they may form, which in turn restricts the type of space groups of these crystals. The correspondence is presented in Table 2, which corrects some imprecisions that appeared in the previous literature.

Achiral molecules can produce both achiral and chiral crystal structure. For the latter, the molecular packing needs to be chiral, which requires the presence of screw axes $n_{p}$ with $p \neq n / 2$. This condition is realized only in 28 types of space group, not in all 65 of the Sohncke types of space group, as stated in the previous literature.

Scalemic enantiopure solutions cannot produce achiral crystal structures; their crystal structures are chiral and crystallize in Sohncke-type space groups.

Scalemic enantioenriched solutions can give achiral crystal structures only in the form of type I (disordered) solid solutions. Ordered crystal structures are either scalemic crystals, in which the chirality comes from the prevalence of one enantiomer over the other, or scalemic conglomerates, in which 
each of the two types of domains contains only one enantiomer and the two types of domains span unequal volumes. In both cases, the space group is of Sohncke type.

Racemic solutions (racemates) can give both achiral and chiral crystal structures. Achiral crystal structures are realized as racemic crystals, in non-Sohncke types of space group, or as kryptoracemates, in Sohncke types of space group. The latter are much rarer and the restriction on $Z^{\prime}>1$ assumed in the past is unnecessary, as shown by the two isostructural examples violating it. Chiral crystal structures are realized in racemic conglomerates, where the two enantiomers concentrate in different domains, each crystallizing in a Sohncke-type space group. The volume spanned by the two types of domain is equal.

Flack (2003) included in his classification 'the counterintuitive case of a chiral crystal structure formed from a racemate'. He justified this assumption by making the hypothesis that any isometry of the second kind relating the two enantiomers 'is not part of the symmetry operations of the space group but rather a 'local' or 'pseudo-symmetry' operation'. This description clearly corresponds to the case of kryptoracemates. The resulting space group is of Sohncke type but the crystal structure itself is not chiral.

\section{References}

Aroyo, M. I., Perez-Mato, J. M., Capillas, C., Kroumova, E., Ivantchev, S., Madariaga, G., Kirov, A. \& Wondratschek, H. (2006). Z. Kristallogr. 221, 15-27.

Bernal, I. (1995). ACA Annual Meeting, Montreal, Quebec, Canada, Abstract 4a.I.e.

Bernal, I. \& Watkins, S. (2015). Acta Cryst. C71, 216-221.
Bishop, R. \& Scudder, M. L. (2009). Cryst. Growth Des. 9, 2890-2894. Brewster, J. H. (1992). Chem. Eng. News, 70(20), 3.

Capillas, C., Tasci, E. S., de la Flor, G., Orobengoa, D., Perez-Mato, J. M. \& Aroyo, M. I. (2011). Z. Kristallogr. 226, 186-196.

Clevers, S. \& Coquerel, G. (2020). CrystEngComm, 22, 7407-7419.

Eliel, E. L. (1997). Chirality, 9, 428-430.

Fábián, L. \& Brock, C. P. (2010). Acta Cryst. B66, 94-103.

Fischer, W., Burlaff, H., Hellner, E. \& Donnay, J. D. H. (1973). Space Groups and Lattice Complexes. Washington, D.C.: US Department of Commerce/National Bureau of Standards.

Flack, H. D. (1983). Acta Cryst. A39, 876-881.

Flack, H. D. (2003). Helv. Chim. Acta, 86, 905-921.

Glazer, A. M. (2018). J. Appl. Cryst. 51, 915-918.

Hauser, A., Maeder, M., Robinson, W. T., Murugesan, R. \& Ferguson, J. (1987). Inorg. Chem. 26, 1331-1338.

Heathcock, C. H. (1991). Chem. Eng. News, 69(5), 3.

IUPAC (1997). Compendium of Chemical Terminology, 2nd ed., compiled by A. D. McNaught \& A. Wilkinson. Oxford: Blackwell Scientific Publications.

Kousaka, Y., Ohsumi, H., Komesu, T., Arima, T., Takata, M., Sakai, S., Akita, M., Inoue, K., Yokobori, T., Nakao, Y., Kaya, E. \& Akimitsu, J. (2009). J. Phys. Soc. Jpn, 78, 123601.

Montoya, J. A., Mejía, C., Bourdón, R. D., Cruz, E. \& Ágreda, J. (2018). MATCH Commun. Math. Comput. Chem. 80, 311-344.

Morales, G. A. \& Fronczek, F. R. (1996). Acta Cryst. C52, 1266-1268.

Nespolo, M., Aroyo, M. I. \& Souvignier, B. (2018). J. Appl. Cryst. 51, 1481-1491.

Nespolo, M., Souvignier, B. \& Litvin, D. B. (2008). Z. Kristallogr. 223, 605-606.

Neugebauer, P., Triebl, A. \& Gruber-Woelfler, H. (2021). J. Flow Chem. https://doi.org/10.1007/s41981-021-00173-2.

Rekis, T. (2020). Acta Cryst. B76, 307-315.

Rekis, T. \& Bērziňš, A. (2018). CrystEngComm, 20, 6909-6918.

Wei, S., Mauksch, M. \& Tsogoeva, S. B. (2009). Chem. Eur. J. 15, 10255-10262.

Zorkii, P. M. (1978). Acta Cryst. A34, S1-S2. 